\title{
Survey on EMI: Privacy-Preserving Public Auditing for Regenerating-Code-Based Cloud Storage
}

\author{
Shilpa Singh ${ }^{1}$, Padmavathi B. ${ }^{2}$ \\ ${ }^{1}$ Department of Computer Engineering, G H Raisoni College of Engineering and Management \\ ${ }^{2}$ Professor, Department of Computer Engineering, G H Raisoni College of Engineering and Management
}

\begin{abstract}
To protect outsourced knowledge in cloud storage against corruptions, adding fault tolerance to cloud storage along with knowledge integrity checking and failure reparation becomes critical. Recently, create codes have gained quality due to their lower repair information measure whereas providing fault tolerance. Existing remote checking strategies for regenerating-coded knowledge only give non-public auditing, requiring knowledge house owners to continuously stay on-line and handle auditing, similarly as repairing, which is sometimes impractical. During this paper, we have a tendency to propose a public auditing theme for the regenerating-code-based cloud storage. To solve the regeneration drawback of unsuccessful authenticators in the absence of knowledge house owners, we have a tendency to introduce a proxy, which is privileged to regenerate the authenticators, into the normal public auditing system model. Moreover, we have a tendency to style a unique public verifiable appraiser that is generated by a few of keys and can be regenerated exploitation partial keys. Thus, our theme will completely unleash knowledge house owners from on-line burden. Additionally, we randomise the code coefficients with a pseudorandom operateto preserve knowledge privacy. Intensive security analysis shows that our theme is demonstrable secure beneath random oracle modeland experimental analysis indicates that our theme is very efficient and might be feasibly integrated into the regenerating code-based cloud storage.
\end{abstract}

Keywords: keys, codes

\section{Introduction}

Cloud storage is currently gaining quality as a result of it offers a versatile on-demand information outsourcing service with appealing benefits: relief of the burden for storage management, universal information access with location independence, and dodging of cost on hardware, software, and personal maintenances, etc., [1]. still, this new paradigm of information hosting service additionally brings new security threats toward users information, therefore creating people or enterprisers still feel hesitant. It is noted that information homeowners lose final management over the fate of their outsourced data; therefore, the correctness, handiness and integrity of the information square measure being place in danger. On the one hand, the cloud service is typically featured with a broad vary of internal/external adversaries, UN agency would maliciously delete or corrupt users' data; on the opposite hand, the cloud serviceproviders might act deceitfully, trying to cover knowledge loss or corruption and claiming that the files are still properly hold on within the cloud for name or financial reasons. Thus itmakes nice sense for users to implement associate degree economical protocol to perform periodical verifications of their outsourced knowledge to confirm that the cloud so maintains their knowledge properly. Several mechanisms addressing the integrity of outsourced knowledge while not a neighbourhood copy are projected beneath totally different system and security models up to currently. The foremost vital work among these studies are the PDP (provable knowledge possession) model and POR (proof of irretrievability) model that were originally projected for the single-server situation byAteniese et al. [2] and Juels ET. al. [3], severally. Considering that files are sometimes stripy and redundantly hold on across multi-servers or multiclouds, [4]-[10] explore integrity verification schemes appropriate for such multi-servers or multi-clouds setting with totally different redundancy schemes, like replication, erasure codes, and, a lot of recently, makecodes. During this paper, we tend to concentrate on the integrity verification downside in regenerating-code-based cloud storage, particularly with the practical repair strategy [11]. Similar studies areperformed by Bo subgenus Chen et al. [7] and $\mathrm{H}$. Chen el al. [8] one by one and severally. [7] Extended the single-server CPOR scheme (private version in [10]) to the regeneratingcode-scenario; [8] designed and enforced an information integrity protection (DIP) theme for FMSR [11]-based cloud storage and therefore the theme is customized to the thin-cloud setting1. However,both of them are designed for personal audit, solely the information owner is allowed to verify the integrity and repair the faulty servers. Considering the big size of the outsourced knowledge and therefore the user's unnatural resource capability, the tasks of auditing and reparation within the cloud may be formidable and valuable for the users [13]. The overhead of mistreatment cloud storage ought tobe reduced the maximum amount as potential specified a user doesn't have to be compelled to perform too several operations to their outsourced knowledge (in extra to retrieving it) [12]. Specially, users might notwant to travel through the quality in confirming and reparation. The auditing schemes in [7], [8] imply the matter that users have to be compelled to forever keep on-line, which can impede its adoption inpractice, particularly for long-run depository storage.

To fully make sure the information integrity and save the users' computation resources moreover as on-line burden, we tend to propose a public auditing theme for the regenerating-code-based cloud storage, during which the integrity checking and regeneration (of unsuccessful information blocks and authenticators) area unit enforced by a third-party auditor and a semi-trusted proxy one by one on behalf of the info owner. Rather than directly adapting the prevailing public auditing theme [11] to the multi-server

\section{Volume 4 Issue 12, December 2015}




\section{International Journal of Science and Research (IJSR) \\ ISSN (Online): 2319-7064 \\ Index Copernicus Value (2013): 6.14 | Impact Factor (2014): 5.611}

setting, we tend to style a unique critic, that is additional applicable for create codes. Besides, we tend to "encrypt" the coefficients to safeguard information privacy against the auditor, that is additional light-weight than applying the proof blind technique in and information blind technique in [12]. Many challenges and threats impromptu arise in our new system model with a proxy (Section II-C), and security analysis shows that our theme works well with these issues

\section{Literature Survey}

1. A. Fox, R. Griffith, A. Joseph, R. Katz, A. Konwinski, G. Lee, D. Patterson, A. Rabkin, and I. Stoica, "Above the clouds: A Berkeley view of cloud computing," Dept. Electrical Eng. and Computer. Sciences,University of California, Berkeley, Rep. UCB/EECS, vol. 28, p. 13, 2009. Cloud Computing, the long-held dream of computing as a utility, has the potential to transform a large part of the IT industry, making software even more attractive as a service and shaping the way IT hardware is designed and purchased. Developers with innovative ideas for new Internet services no longer require the large capital outlays in hardware to deploy their service or the human expense to operate it. They need not be concerned about over-provisioning for a service whose popularity does not meet their predictions, thus wasting costly resources, or under-provisioning for one that becomes wildly popular, thus missing potential customers and revenue. Moreover, companies with large batch-oriented tasks can get results as quickly as their programs can scale, since using 1000 servers for one hour costs no more than using one server for 1000 hours. This elasticity of resources, without paying a premium for large scale, is unprecedented in the history of IT.

2.G. Ateniese, R. Burns, R. Curtmola, J. Herring, L. Kissner, Z. Peterson, and D. Song, "Provable data possession at untrusted stores," in Proceedingsof the 14th ACM Conference on Computer and Communications Security, ser. CCS '07. New York, NY, USA: ACM, 2007, pp. 598-609.

We introduce a model for provable data possession (PDP) that allows a client that has stored data at an untrusted server to verify that the server possesses the original data without retrieving it. The model generates probabilistic proofs of possession by sampling random sets of blocks from the server, which drastically reduces $\mathrm{I} / \mathrm{O}$ costs. The client maintains a constant amount of metadata to verify the proof. The challenge/response protocol transmits a small, constant amount of data, which minimizes network communication. Thus, the PDP model for remote data checking supports large data sets in widely-distributed storage systems. We present two provably-secure PDP schemes that are more efficient than previous solutions, even when compared with schemes that achieve weaker guarantees. In particular, the overhead at the server is low (or even constant), as opposed to linear in the size of the data. Experiments using our implementation verify the practicality of PDP and reveal that the performance of PDP is bounded by disk I/O and not by cryptographic computation

3.A. Juels and B. S. Kaliski Jr, "Pors: Proofs of irretrievability for largefiles," in Proceedings of the 14th ACM conference on Computer andcommunications security. ACM, 2007, pp. 584-597.
Cloud computing has been envisioned as the paramount solution to the rising storage device costs of IT Enterprises. With the high costs of data storage devices as well as the rapid rate at which data is being generated it proves expensive for enterprises or individual users to frequently update their hardware. Apart from reduction in storage costs data outsourcing to the cloud also helps in reducing the maintenance. Cloud storage moves the user's data to large data centres, which are remotely located, on which user does not have any control. However, this unique feature of the cloud rises many new security challenges which need to be clearly understood and resolved. One of the important concerns that need to be addressed is to assure the data integrity to customer. I.e. correctness of his data in the cloud. As the data is physically not accessible to the user the cloud should provide a way for the user to check if the integrity of his data is maintained or is compromised. In thispaper a scheme is proposed which gives a proof of data integrity in the cloud which the customer can employ to check the correctness of his data in the cloud. This proof can be agreed upon by both the cloud and the customer and can be incorporated in the Service level agreement (SLA). This scheme ensures that the storage at the client side is minimal which will be beneficial for the organization

4. R. Curtmola, O. Khan, R. Burns, and G. Ateniese, "Mrpdp: Multiplereplicaprovable data possession," in Distributed Computing Systems, 2008. ICDCS'08. The 28th International Conference on. IEEE, 2008, pp. 411-420.

Many storage systems rely on replication to increase the availability and durability of data on untrusted storage systems. At present, such storage systems provide no strong evidence that multiple copies of the data are actually stored. Storage servers can collude to make it look like they are storing many copies of the data, whereas in reality they only store a single copy. We address this shortcoming through multiple-replica provable data possession (MR-PDP): A provably-secure scheme that allows a client that stores $t$ replicas of a file in a storage system to verify through a challenge-response protocol that (1) each unique replica can be produced at the time of the challenge and that (2) the storage system uses $t$ times the storage required to store a single replica. MR-PDP extends previous work on data possession proofs for a single copy of a file in a client/server storage system (Ateniese et al., 2007). Using MR-PDP to store $t$ replicas is computationally much more efficient than using a single-replica PDP scheme to store $t$ separate, unrelated files (e.g., by encrypting each file separately prior to storing it). Another advantage of MR-PDP is that it can generate further replicas on demand, at little expense, when some of the existing replicas fail.

\section{Proposed Approach Framework and Design}

\section{Cloud Server}

A native Cloudthat provides priced rife storage services square measure been created in this module. The users will transfer their information within the cloud. This module may be developed wherever the cloud storage can becreated secure. The cloud is not absolutely honourable by users since the CSPs square measure terribly possible to be outside of the cloud users' trustworthy domain. The same as 


\section{International Journal of Science and Research (IJSR) \\ ISSN (Online): 2319-7064}

Index Copernicus Value (2013): 6.14 | Impact Factor (2014): 5.611

that the cloud server is real however curious. That is, the cloud server cannot maliciously delete or modify user information due to the protection of information investigation schemes, however can attempt to learn the content of the hold on information and the identities of cloud users. This primarily implies that the owner (client) of the information moves its information to a 3rd party cloud storage server that square measure speculated to presumptively for a fee really store the information with it and supply it back to the owner whenever needed.

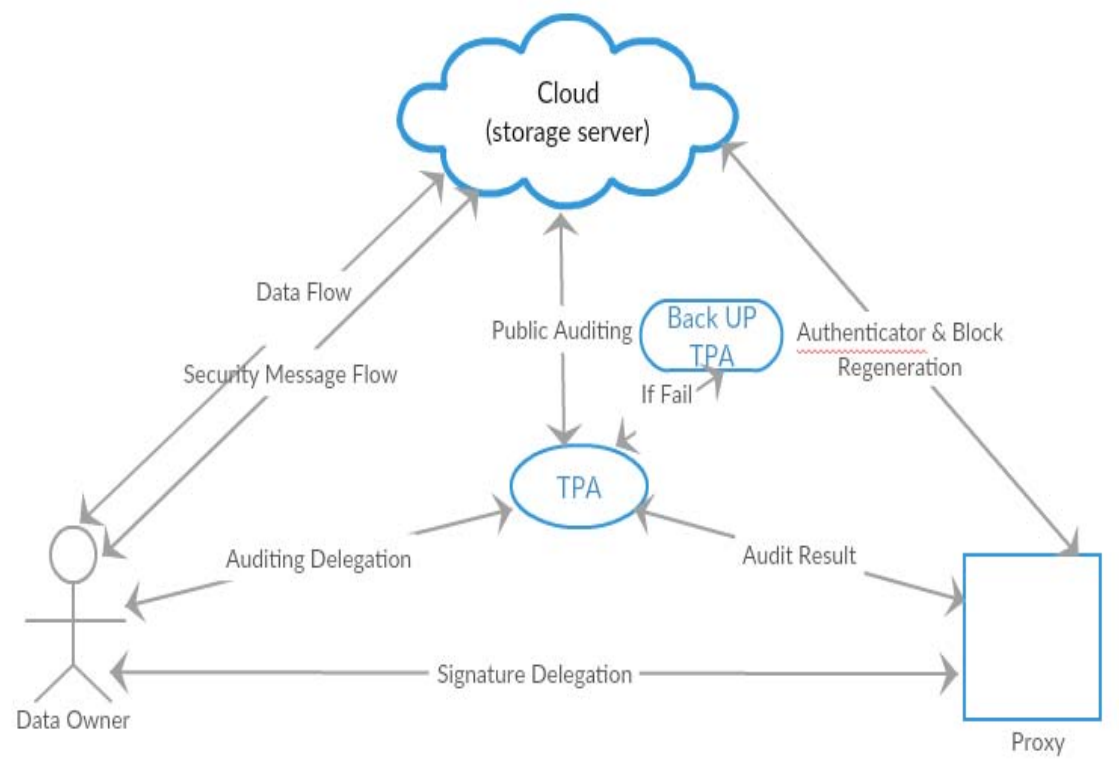

Figure 1: Proposed System Architecture

\section{Proxy Server:}

A proxy agent acts on behalf of information the info the information owner to regenerate authenticators and data blocks on the servers throughout the repair procedure. Notice that the information owner is restricted in machine and storage resources compared to alternative entities and should become off-line when the knowledge transfer procedure. The proxy, UN agency would continuously be on-line, is meant to be rather more powerful than the information owner however less than the cloud servers in terms of computation and memory capability. To save resources as well as the on-line burden doubtless brought by the periodic auditing and accidental repairing, the knowledge house owners resort to the TPA for integrity verification and delegate the reparation to the proxy. Considering that the information owner cannot continuously keep on-line in observe, so as tokeep the storage offered and verifiable when a malicious corruption, we have a tendency to introduce a semi-trusted proxy into the system model and supply a privilege for the proxy to handle the reparation of the coded blocks and authenticators. It generates signature exploitation OAEP primarily based key delegation that provides distinctive non-public and public key for every cluster registered within the cloud. Therefore the users will access the document provided by its own cluster solely. The users will read alternative team's document exploitation non-public key of the opposite teams. If he modifies alternative cluster content he are revoked by the cloud server.

TPA:

TPA is trusty and its audit result's unbiased for each knowledge homeowners and cloud servers; and a proxy agent, UN agency is semi-trusted and acts on behalf of the knowledge information $\}$ owner to regenerate authenticators and data blocks on the unsuccessful servers throughout the repair procedure. Notice that the info owner is restricted in procedure and storage resources compared to alternative entities and will becomes off-line even once the info transfer procedure. The proxy, who would always be on-line, is meant to be far more powerful than the info owner however but the cloud servers in terms of computation and memory capability. to avoid wasting resources likewise because the on-line burden probably brought by the periodic auditing and accidental repairing, the info homeowners resort to the TPA for integrity verification and delegate the reparation to the proxy.

\section{Auditing Scheme}

$\operatorname{KeyGen}(1 \kappa) \rightarrow(\mathrm{pk}, \mathrm{SK}):$ This polynomial-time algorithm is run by the data owner to initialize its public and secret parameters by taking a security parameter $\kappa$ as input

Delegation $(\mathbf{S K}) \rightarrow(\mathrm{x})$ : This algorithm represents the Interaction between the data owner and proxy. The data ownerdelivers partial secret key $\mathrm{x}$ to the proxy through a secureapproach.

Sig And Block Gen (sk, F): This polynomialtime algorithm is run by the data owner and takes the secretparameter sk and the original file $\mathrm{F}$ as input, and then outputsa coded block set $\varnothing$, an authenticator set $\psi$ and a file tag t.

Challenge(Finfo) $\rightarrow(\mathrm{C})$ : This algorithm is performed by the TPA with the information of the file Finfo as input and a challenge $\mathrm{C}$ as output.

\section{Volume 4 Issue 12, December 2015}




\section{International Journal of Science and Research (IJSR) \\ ISSN (Online): 2319-7064}

Index Copernicus Value (2013): 6.14 | Impact Factor (2014): 5.611

$\operatorname{ProofGen}(\mathrm{C}, \phi, \Psi) \rightarrow(\mathrm{P})$ : This algorithm is run by each cloud server with input challenge $C$, coded block set and authenticator set $\phi$ then it outputs a proof $\mathrm{P}$.

Verify $(\mathrm{P}, \mathrm{pk}, \mathrm{C}) \rightarrow(0,1)$ : This algorithm is run by TPA immediately after a proof is received. Taking the proof $\mathrm{P}$, public parameter pk and the corresponding challenge $\mathrm{C}$ as input, it outputs 1 if the verification passed and 0 otherwise.

\subsection{Hardware and Software Configuration}

\section{Hardware Requirements:}

- Processor : Pentium IV 2.6 GHz

- RAM :512 MB DDR RAM

- Hard Disk : 20 GB

\section{Software Requirements:}

- Front End : Java

- Tools Used : NetBeans

- Operating System : Windows 7/8

- Database : MySQL

\section{Conclusion and Future Work}

We propose a public auditing scheme for the regeneratingcode-based cloud storage system, where the data owners are privileged to delegate TPA for their data validity checking. To protect the original data privacy against the TPA, we randomize the coefficients in the beginning rather than applying the blind technique during the auditing process.

\section{References}

[1] A. Fox, R. Griffith, A. Joseph, R. Katz, A. Konwinski, G. Lee,D. Patterson, A. Rabkin, and I. Stoica, "Above the clouds: A Berkeley view of cloud computing," Dept. Electrical Eng. and Compute. Sciences,University of California, Berkeley, Rep. UCB/EECS, vol. 28, p. 13, 2009.

[2] G. Ateniese, R. Burns, R. Curtmola, J. Herring, L. Kissner, Z. Peterson, and D. Song, "Provable data possession at untrusted stores," in Proceedingsof the 14th ACM Conference on Computer and CommunicationsSecurity, ser. CCS '07. New York, NY, USA: ACM, 2007, pp. 598-609.

[3] A. Juels and B. S. Kaliski Jr, "Pors: Proofs of irretrievability for largefiles," in Proceedings of the 14th ACM conference on Computer andcommunications security. ACM, 2007, pp. 584-597.

[4] R. Curtmola, O. Khan, R. Burns, and G. Ateniese, "Mrpdp: Multiple replicaprovable data possession," in Distributed Computing Systems,2008. ICDCS'08. The 28th International Conference on. IEEE, 2008,pp. 411420.

[5] K. D. Bowers, A. Juels, and A. Oprea, "Hail: a highavailability andintegrity layer for cloud storage," in Proceedings of the 16th ACMconference on Computer and communications security. ACM, 2009,pp. 187-198.

[6] J. He, Y. Zhang, G. Huang, Y. Shi, and J. Cao, "Distributed datapossession checking for securing multiple replicas in geographically dispersedclouds,' Journal of Computer and System Sciences, vol. 78,no. 5, pp. 1345-1358, 2012.

[7] B. Chen, R. Curtmola, G. Ateniese, and R. Burns, "Remote datachecking for network coding-based distributed storage systems," inProceedings of the 2010 ACM workshop on Cloud computing securityworkshop. ACM, 2010, pp. 31-42.

[8] H. Chen and P. Lee, "Enabling data integrity protection in regenerating coding-based cloud storage: Theory and implementation," Parallel andDistributed Systems, IEEE Transactions on, vol. 25, no. 2, pp. 407-416,Feb 2014.

[9] K. Yang and X. Jia, "An efficient and secure dynamic auditing protocolfor data storage in cloud computing," Parallel and Distributed Systems,IEEE Transactions on, vol. 24, no. 9, pp. 1717-1726, 2013.

[10] Y. Zhu, H. Hu, G.-J. Ahn, and M. Yu, "Cooperative provable datapossession for integrity verification in multi-cloud storage," Parallel and Distributed Systems, IEEE Transactions on, vol. 23, no. 12, pp. 2231-2244, 2012.

[11]A. G. Dimakis, K. Ramchandran, Y. Wu, and C. Suh, "A survey onnetwork codes for distributed storage," Proceedings of the IEEE, vol. 99, 